\title{
POR QUÉ SE PRODUCE LA VIOLENCIA ESCOLAR Y CÓMO PREVENIRLA
}

\section{María José Díaz-Aguado*}

SíNTESIS: En este artículo se analizan, desde una perspectiva ecológica, las condiciones de riesgo y de protección de la violencia escolar, prestando una especial atención a la serie de estudios que la autora ha dirigido sobre este tema con adolescentes. Por otra parte, los resultados que reflejan la especial relevancia que el modelo dominio-sumisión tiene en los agresores, el aislamiento que caracteriza a la situación de las víctimas, así como tres condiciones de riesgo estrechamente asociadas con el currículum oculto de la escuela tradicional respecto a la violencia: la tendencia a minimizarla, su pasividad, y la ausencia de un tratamiento adecuado respecto a la diversidad existente en el alumnado. A partir de ellos se propone desarrollar la prevención a través de innovaciones que ayuden a redefinir la función del profesorado y su disponibilidad para ayudar, el papel del alumno, las relaciones que se establecen entre compañeros mediante el aprendizaje cooperativo y del currículum de la no-violencia, y la antítesis del modelo de dominio-sumisión en el que se basa la violencia. Las respuestas obtenidas en un programa evaluado con 783 adolescentes confirman la eficacia y la viabilidad de dichos componentes en la prevención de la violencia entre iguales, así como en la mejora de las relaciones con el profesorado y en las propias del aprendizaje.

SÍNTESIS: Neste artigo são analisadas, sob uma perspectiva ecológica, as condições de risco e de proteção da violência escolar, prestando especial atenção à série de estudos que a autora tem dirigido sobre este tema com adolescentes. Por outro lado, os resultados que refletem a especial relevância que o modelo domínio-submissão tem nos agressores, o isolamento que caracteriza a situação das vítimas, assim como três condições de risco estreitamente associadas ao currículo oculto da escola tradicional em relação à violência: a tendência a minimizá-la, sua passividade, e a ausência de um tratamento adequado com respeito à diversidade existente no alunado. A partir disso, propõe-se a desenvolver a prevenção através das inovações que ajudem a redefinir a função do professorado e sua disponibilidade para ajudar, o papel do aluno, as relações que se estabelecem entre companhei ros mediante a aprendizagem

España.

* Catedrática de Psicología de la Educación, Universidad Complutense, Madrid, 
cooperativa e do currículo da não-violência, e a antítese do modelo de domínio-submissão, no qual se baseia a violência. As respostas obtidas em um programa avaliado com 783 adolescentes confirmam a eficácia e a viabilidade de tais componentes na prevenção da violência entre iguais, assim como na melhora não só das relações com o professorado como também na aprendizagem.

\section{INTRODUCCIÓN}

Las propuestas y los resultados que aquí se presentan se sitúan dentro de una larga serie de investigaciones sobre cómo prevenir la violencia desde la educación [Díaz-Aguado (dir.), 1992, 1996, 2002, 2004]. La última, a la que corresponden los datos específicos que sobre el acoso entre escolares se incluyen en este texto, ha sido publicada recientemente con el título Prevención de la violencia y la lucha contra la exclusión desde la adolescencia [Díaz-Aguado (dir.), 2004] [. Es la continuación de dos series anteriores: Programas de educación para la tolerancia y prevención de la violencia en los jóvenes, iniciada en 1994 mediante un convenio de colaboración entre la U niversidad Complutense, el Ministerio de Educación y Ciencia y el Instituto Nacional de la Juventud, que la publicó en cuatro libros y dos vídeos [Díaz-Aguado (dir. ), 1996], y Prevenir la violencia contra las mujeres construyendo la igualdad (Díaz-Aguado, 2002; Díaz-Aguado y Martínez Arias, 2001) publicada por el Instituto de la Mujer.

\section{POR QUÉ SE PRODUCE LA VIOLENCIA ESCOLAR}

\subsection{CONDICIONES DE RIESGO Y DE PROTECCIÓN DESDE UN ENFOQUE ECOLÓGICO-EVOLUTIVO}

Para prevenir la violencia escolar conviene tener en cuenta que las condiciones de riesgo y de protección que sobre ella influyen son múltiples y complejas. Además, que es preciso analizarlas desde una

\footnotetext{
${ }^{1}$ Estos programas han sido publicados por el Instituto de la J uventud en tres libros y un vídeo con el título Prevención de la violencia y lucha contra la exclusión desde la adolescencia, que pueden pedir de forma gratuita las instituciones que trabajan en este ámbito, explicando el objetivo de la solicitud a: foroinjuve@mtas.es. Los tres libros están disponibles en Internet en la dirección mtas. es/injuve/novedades/prevenciónviolencia.htm Y los tres programas de vídeo en la videoteca virtual de atei, a través de la dirección www.atei.es, dentro de los «Programas de atención a la diversidad y educación en valores en la escuela actual». También a través de: http://mariajosediaz-aguado.tk
} 
perspectiva evolutiva ya distintos niveles, incluyendo, junto a la interacción que el alumnado establece en la escuela, la que existe en la familia, la calidad de la colaboración entre ambos contextos, la influencia de los medios de comunicación, o el conjunto de creencias, de valores y de estructuras de la sociedad de la que forman parte. Cuando se analiza cada caso de violencia escolar desde esta perspectiva, suelen encontrarse incontables clases de riesgo y escasas o nulas condiciones protectoras en cada nivel y desde edades tempranas. Entre las categorías de riesgo detectadas en los estudios científicos, y que suelen verse reflejadas en la mayoría de los casos de violencia escolar, cabe destacar: la exclusión social o el sentimiento de exclusión, la ausencia de límites, la exposición a la violencia a través de los medios de comunicación, la integración en bandas identificadas con la violencia, la facilidad para disponer de armas, y la justificación de la violencia en la sociedad en la que dichas circunstancias se producen. $Y$ faltan condiciones que hubieran podido proteger de tales riesgos, como modelos sociales positivos y solidarios, colaboración entre la familia y la escuela, contextos de ocio y de grupos de pertenencia constructivos, 0 adultos disponibles y dispuestos a ayudar. Para mejorar la convivencia escolar y para prevenir la violencia, se debería intervenir lo antes posible (sin esperar a las graves manifestaciones que suelen alertar sobre dicha necesidad) y en todos estos niveles, desde una doble perspectiva:

- La perspectiva evolutiva, analizando las condiciones de riesgo y de protección que pueden existir en cada momento evolutivo, en función de las tareas y de las habilidades vitales básicas. La comprensión de dichas tareas es de gran utilidad para adecuar la intervención a cada edad, ayudando a desarrollar las habilidades críticas de ese período, reforzando los logros conseguidos y compensando las deficiencias que se hayan podido producir en edades anteriores. Debido a esto, se propone que la prevención debe fortalecer cuatro capacidades fundamentales que permitan al alumno/a: establecer vínculos de calidad en diversos contextos; ser eficaz en situaciones de estudio-trabajo, movilizando la energía y el esfuerzo precisos para ello, y obteniendo el reconocimiento social necesario; integrarse en grupos de iguales constructivos, resistiendo presiones inadecuadas; y desarrollar una identidad propia y diferenciada que le ayude a encontrar su lugar en el mundo y le permita apropiarse de su futuro. 
- La perspectiva ecológica, que trata de las condiciones de riesgo y de protección en los complejos niveles de la interacción individuo-ambiente, a partir de la cual se pueda diseñar la prevención con actividades destinadas a optimizar tanto el ambiente como la representación que de él y de sus posibilidades tiene el alumnado, incluyendo los escenarios en los que transcurre su vida (escolar, familiar, de ocio...), las conexiones entre dichos escenarios, los medios de comunicación, y el conjunto de las creencias y de las estructuras de la sociedad.

\subsection{EL ACOSO ENTRE IGUALES}

En la última década se ha incrementado mucho la toma de conciencia respecto a un problema que es tan viejo y generalizado como la propia escuela tradicional: el acoso entre iguales. Los resultados obtenidos en los estudios científicos realizados sobre su incidencia reflejan que, a lo largo de su vida en la escuela, todos los estudiantes parecen haber tenido contacto con la violencia entre iguales, ya sea como víctimas, ya sea como agresores, ya sea como espectadores, siendo esta última la situación más frecuente.

\section{Características de los agresores. El modelo dominio- sumisión como origen de la violencia}

Entre las características más frecuentes observadas en los alumnos que acosan a sus compañeros, destacan las siguientes (Olweus, 1993 Pellegrini, Bartini y Brooks, 1999; Salmivalli y otros, 1996; Schwartz, Dodge, Pettit y Bates, 1997): una situación social negativa, aunque cuentan con algunos amigos que les siguen en su conducta violenta; una acentuada tendencia a abusar de su fuerza (suelen ser físicamente más fornidos que los demás); son impulsivos, con escasas habilidades sociales, con baja tolerancia a la frustración, y con dificultad para cumplir normas; unas relaciones negativas con relación a los adultos y un bajo rendimiento, problemas que se incrementan con la edad; no son muy autocríticos, por lo que cabe considerar el hecho observado en varias investigaciones al intentar evaluar la autoestima de los agresores y encontrarla media o incluso alta. Entre los principales antecedentes familiares, suelen destacarse: la ausencia de una relación afectiva cálida y segura por parte de los padres, sobre todo de la madre, que manifiesta 
actitudes negativas o escasa disponibilidad para atender al niño; y fuertes dificultades para enseñar a respetar límites, combinando la permisividad ante conductas antisociales con el frecuente empleo de métodos autoritarios y coercitivos, utilizando en muchos casos el castigo corporal.

En el estudio que realizamos con adolescentes (Díaz-Aguado, Martínez Arias y Martín Seoane, 2004) se refleja, así mismo, que los agresores tienen menor disponibilidad de estrategias no violentas para la resolución de conflictos, detectando, por otra parte, las siguientes carencias en torno a las cuales convendría también orientar la prevención de este problema:

- Están de acuerdo con las creencias que llevan a justificar la violencia y la intolerancia en distintos tipos de relaciones, incluidas las que se producen entre pares, manifestándose igualmente como más racistas, xenófobos y sexistas, es decir, que tienden a identificarse con un modelo social basado en el dominio de los unos y en la sumisión de los otros.

- Tienen dificultades para colocarse en el lugar de los demás. Su razonamiento moral es primitivo si se compara con el de sus compañeros, siendo más frecuente entre los agresores la identificación de la justicia con «hacer a los demás lo que te hacen a ti o con lo que crees que te hacen», orientación que puede explicar su tendencia a vengar ofensas reales 0 supuestas. Y comulgan con una serie de conceptos relacionados con el acoso escolar, como los de chivato y cobarde, que utilizan para justificarlo y para mantener la conspiración de silencio que lo perpetúa.

- Están menos satisfechos que sus compañeros con su aprendizaje escolar y con las relaciones que establecen con los profesores. En ese sentido, parece existir una estrecha relación entre la tendencia a acosar a los condiscípulos y la de hacerlo con el profesorado, y entre ambos problemas y la percepción de haber sufrido tal tipo de situaciones en la relación con los profesores (Mendoza, 2005).

- Son considerados por sus compañeros como intolerantes y arrogantes, pero al mismo tiempo se sienten fracasados. EI conjunto de características en las que destacan sugiere que 
cuentan con iguales que les siguen en sus agresiones, formando grupos con disposición a la violencia, en los que se integrarían individuos que no han tenido muchas oportunidades de protagonismo positivo en el sistema escolar.

- Su frecuencia es mayor en la adolescencia temprana (13-15 años), en los cursos de educación secundaria obligatoria, en los que se experimenta una mayor dependencia del grupo de compañeros, en aquellos que suelen resultar más difíciles para el profesorado de secundaria en el contexto evaluado en este estudio [Díaz-Aguado (dir.), 2004].

Los resultados expuestos ponen de manifiesto la importancia que tiene erradicar situaciones de exclusión desde las primeras etapas educativas, y favorecer la identificación de los adolescentes con los valores de respeto mutuo, de empatía y de no violencia para prevenir el acoso entre escolares.

\section{La situación de las víctimas}

Entre los estudiantes que son víctimas de acoso suelen diferenciarse dos situaciones (Díaz-Aguado, Martínez Arias y Martín Seoane, 2004; Olweus, 1993; Pellegrini, Bartini y Brooks, 1999; Salmivalli y otros, 1996; Schwartz, Dodge, Pettit y Bates, 1997; Smith y otros, 2004):

- La víctima típica, o víctima pasiva, que se caracteriza por una situación social de aislamiento, en relación con la cual cabe considerar su escasa asertividad y su dificultad de comunicación; una conducta muy pasiva; miedo ante la violencia y manifestación de vulnerabilidad (de no poder defenderse antela intimidación); acusada ansiedad, inseguridad y baja autoestima, características que cabe relacionar con la tendencia observada en algunas investigaciones en las víctimas pasivas acerca de culpabilizarse ellas mismas de su situación y hasta de negarla, debido quizás a que la consideran como más vergonzosa de lo que lo hacen con la suya los agresores (que a veces parecen estar orgullosos de serlo).

- La victima activa, que se caracteriza por una situación social de aislamiento y de aguda impopularidad, Ilegando a encontrarse entre los alumnos con mayor rechazo por parte sus compañeros (superior al que tienen los agresores y las 
víctimas pasivas), situación que podría estar en el origen de su selección como víctimas, aunque, como en el caso de las anteriores, también podría agravarse con la victimización; una tendencia excesiva e impulsiva a actuar, a intervenir sin llegar a poder elegir la conducta que puede resultar más adecuada a cada situación; con problemas de concentración (llegando incluso, en algunos casos, a la hiperactividad), y con cierta disponibilidad a reaccionar mediante conductas agresivas e irritantes. Dichas características han hecho que, en ocasiones, este tipo de víctimas sea considerada como «provocadora», asociación que convendría evitar para prevenir la frecuente tendencia a culpar a la víctima que suele existir respecto a cualquier tipo de violencia, también en la escolar. La situación de las víctimas activas es la que parece tener un peor pronóstico a largo plazo.

Una significación especial respecto a la prevención de la victimización tienen los resultados obtenidos en algunos estudios (aunque poco mencionados en el conjunto de la investigación), según los cuales el riesgo de ser víctima de acoso se incrementa por el hecho de pertenecer a una minoría étnica en situación de desventaja (DíazAguado, 1992; Troyna y Hatcher, 1992); por presentar dificultades de aprendizaje en aulas ordinarias (Nabuzoka y Smith, 1993); por manifestar complicaciones de expresión verbal (Hugh-J ones y Smith (1999); o por la que puede existir entre los chicos que contrarían el estereotipo sexista tradicional (Rivers, 1999; Young y Sweeting, 2004).

¿Cómo interpretar los resultados obtenidos sobre las características de las víctimas? ¿Pueden ser considerados como meros problemas individuales, $y$, por tanto, superables a través del entrenamiento de las víctimas en habilidades para salir de dicha situación? o ¿exigen ser reconocidos también como problemas de un sistema escolar en el que pueden reproducirse los distintos tipos de exclusión y de acoso (racista, sexista...) que tienen lugar en el conjunto de la sociedad? La respuesta que se dé a estos interrogantes tiene una gran importancia, porque de ella dependerá la eficacia de las intervenciones que se lleven a cabo, así como la posibilidad de contribuir a contrarrestar la frecuente tendencia que se produce en todo tipo de violencia (incluida la escolar) a justificar el acoso culpabilizando a la víctima, que suele activarse no sólo en los agresores sino también en las propias víctimas y en las personas de su entorno. Tal tendencia está muy relacionada con la necesidad de creer que el mundo es justo (Lerner, 1980), y con la de confiar en que los 
graves acontecimientos que observamos a nuestro alrededor no nos sucederán. Para no contribuir a dicha tendencia, y sí a la de su superación, es preciso cuidar de forma especial las descripciones psicológicas acerca de la situación de la víctima, enfatizando los cambios que es necesario ll evar a cabo en la escuel a para prevenir la violencia, evitando describir las características individuales que incrementen el riesgo con términos que puedan activar la tendencia a culpar a la víctima.

\section{El papel de los compañeros frente a la exclusión y al acoso}

El estudio del papel de los compañeros que no participan directamente en el acoso está creciendo de forma importante en los últimos años (Cowie, 2000 ; Pellegrini, Bartini y Brooks, 1999; Salmivalli yotros, 1996; 1998), al observarse que suelen estar presentes en la mayoría de las situaciones en las que se produce. Como reflejo de laimportancia que tiene su papel, cabe destacar uno de los resultados que, de manera sistemática, se repite en los estudios sobre el perfil de las víctimas y de los agresores, en los que se observa que las primeras suelen estar muy aisladas, sin amigos, y ser bastante impopulares, sufriendo ambos problemas en mayor medida que los agresores, sobre todo entre las víctimas activas. Para explicarlo conviene tener en cuenta que la falta de amigos puede originar el inicio de la victimización, y que ésta puede hacer que disminuya aún más la popularidad de quien la sufre y de que aumente su aislamiento (Baker, 1998; Cowie, 2000; Pellegrini y otros., 1999; Salmivalli y otros, 1996; Smith y otros., 2004). Por otra parte, también se ha observado que tener amigos y caer bien son circunstancias que protegen contra la víctimización, aunque el carácter protector de los amigos es casi nulo si proceden del grupo de víctimas, debido tal vez a su debilidad a la hora de intervenir. De ahí se desprende que, para prevenir la violencia, es necesario intervenir con el conjunto de la clase, favoreciendo el tipo de relaciones entre compañeros que inhiba su aparición (Salmivalli, 1999).

\section{Características de la escuela tradicional que incrementan el riesgo: incoherencia y currículum oculto respecto a la violencia}

El conjunto de resultados obtenidos sobre el acoso entre iguales, refleja que determinadas características de la escuela tradicional contribuyen a dicho problema y dificultan su superación, como son: 
- La tendencia a minimizar la gravedad de las agresiones entre iguales, considerándolas como inevitables sobre todo entre los chicos, o como problemas que ellos deben aprender a resolver sin que los adultos intervengan, para hacerse más fuertes, para «curtirse». Como reflejo de su extensión actual, cabe destacar que, en nuestro estudio (Díaz-Aguado, Martínez Arias y Martín Seoane, 2004), el 37,6\% de los adolescentes evaluados manifestó su aceptación de la creencia de que «Si no devuelves los gol pes que recibes, los demás pensarán que eres un cobarde», muy de acuerdo con una de las frases que con cierta frecuencia siguen transmitiendo los adultos a los niños: «Si te pegan, pega», en la que se refleja que la incoherencia escolar respecto a esta violencia expresa otra incoherencia existente aún más amplia en el resto de la sociedad en relación con dicho problema.

- El tratamiento tradicionalmente dado a la diversidad, actuando como si no existiera. En función de esto puede explicarse que el hecho de estar en minoría, de ser percibido como diferente, de tener un problema, o de destacar por una cualidad envidiada, incremente la probabilidad de ser elegido como víctima de acoso (a través de motes, de aislamiento...).

- Insuficiencia de la respuesta que la escuela tradicional suele dar cuando se produce la violencia entre escolares, que deja a las víctimas sin la ayuda que necesitarían para salir de la situación, y que suele ser interpretada por los agresores como un apoyo implícito. Esa falta de respuesta está relacionada con la forma tradicional de definir el papel del profesorado, sobre todo en el nivel de la educación secundaria, orientado de forma casi exclusiva a impartir una determinada materia.

En relación con la falta de respuesta de la escuela tradicional respecto a la violencia, cabe interpretar el resultado obtenido en nuestra investigación (Díaz-Aguado, Martínez Arias y Martín Seoane, 2004), según el cual el 34,6\% de los adolescentes evaluados declaró que nunca pediría ayuda al profesorado si sufriera acoso de parte de sus compañeros, aludiendo, para justificarlo, que «los profesores de secundaria están para enseñarte, no para resolver tus problemas», aunque matizan que «sí pedirían ayuda a un profesor que diera confianza». Tales respuestas 
reflejan la necesidad de adaptar el papel del profesorado a la nueva situación para incrementar su eficacia educativa, así como para la ayuda que puedan proporcionar en la prevención de la violencia. Aunque cada día es mayor la disponibilidad del profesorado para acabar con los problemas expuestos, y aunque ello debería contribuir a erradicar la violencia escolar, no siempre sucede así, debido a la dificultad que supone cambiar pautas muy arraigadas en dicha institución, para cuya superación es preciso dotar al profesorado de los medios que lo hagan posible.

\subsection{EXCLUSIÓN ESCOLAR Y VIOLENCIA}

Existen muy pocas investigaciones que relacionen la violencia entre escolares con los procesos de discriminación y de exclusión en la interacción profesor-alumno, tema que comienza a ser conceptualizado como condición de riesgo en los últimos años. Este cambio en su representación puede ayudar a comprender su posible influencia en el maltrato entre escolares, al incrementar situaciones de riesgo (aislamiento, modelo y justificación para el maltrato, deterioro del clima del aula, división del grupo de clase en subgrupos enfrentados, elevado nivel de estrés...), y reducir las condiciones de protección contra la violencia (adultos a los que acudir, contextos y modelos para la resolución pacífica de los conflictos, normas coherentes y contrarias a todo tipo de violencia...).

Como reflejo de la influencia que sobre todo tipo de violencia pueden tener las experiencias de exclusión vividas en la escuela, cabe considerar los resultados obtenidos desde los primeros estudios longitudinales realizados sobre este tema, en los que se ha observado que los individuos que llevaron a cabo acciones antisociales graves en la juventud y en la edad adulta, se diferenciaban desde los 8 años de sus compañeros por las siguientes razones: ser rechazados por sus compañeros de clase; llevarse mal con sus profesores; manifestar hostilidad hacia diversas figuras de autoridad; expresar baja autoestima; tener dificultades para concentrarse, para planificar y para terminar sus tareas; falta de identificación con el sistema escolar; y abandonar prematuramente la escuela (Glueck y Glueck, 1960; Coleman, 1982; Conger y otros, 1965).

Los resultados obtenidos en nuestras investigaciones con estudiantes de secundaria sugieren también que la exclusión social puede estar en el origen de su identificación con la violencia [Díaz-Aguado, 
(dir.), 1996]. En este sentido, una de las evidencias más significativas procede del estudio comparativo realizado para elaborar los Programas de educación para la tolerancia y prevención de la violencia en los jóvenes, llevado a cabo con 601 chicos entre 14 y 20 años de centros de secundaria de Madrid, en el que observamos que los que se identificaban con dicho problema (la violencia) se diferenciaban del resto de sus compañeros y compañeras de clase por razonar sobre situaciones de conflicto entre derechos de forma más primitiva (con mayor absolutismo e individualidad); por justificar la violencia y utilizarla con más frecuencia; por llevarse mal con los profesores; por ser rechazados por los demás de la clase, y por ser percibidos como agresivos, con fuerte necesidad de protagonismo, inmaduros, antipáticos y con problemas para comprender la debilidad de los demás. Este perfil refleja como posibles causas de la violencia la dificultad para sentirse aceptados y reconocidos por la escuela y por el sistema social en el que se incluyen. Tal resultado apoya, una vez más, la importancia que tiene la lucha contra la exclusión que se produce en la escuela para prevenir todo tipo de violencia, y en función de la cual puede explicarse la eficacia de los programas que favorecen la integración en grupos de compañeros constructivos para lograr la desvinculación con los grupos violentos [Díaz-Aguado (dir.), 1996; Hritz y Gabow, 1997].

\subsection{EL PROFESORADO COMO VÍCTIMA}

Las noticias que con cierta frecuencia aparecen en los medios de comunicación nos alertan sobre algunos casos en los que el profesorado, especialmente en secundaria, llega a ser víctima de la violencia de sus alumnos. En este sentido, ¿qué se encuentra en los estudios científicos? En las escasas investigaciones realizadas, se confirma la necesidad de incluirla en los estudios sobre acoso (Terry, 1998) y de evaluarla desde una doble dirección, que permita registrar tanto el maltrato que pudiera existir desde el profesorado hacia el alumnado, estudiado desde hace décadas con otra denominación (tratamiento discriminatorio generalmente), como desde el alumnado hacia el profesorado, problema que parece estar creciendo en los últimos años, y en el que los adolescentes reconocen estar participando con una frecuencia cada vez mayor (Mendoza, 2005).

En la investigación realizada por Terry (1998) con 101 profesores de siete centros de secundaria del norte de Midlands (Reino Unido), se encuentra que el $9.9 \%$ afirma haber sufrido bullying, o haber 
sido acosado por parte de sus alumnos varias veces por semana. Así se confirma la hipótesis según la cual los profesores novatos sufren dicha situación con mayor frecuencia que los que llevan tiempo. Sin embargo, no se confirma que las mujeres la sufran más que los hombres. Respecto a si conocen al gún compañero que sufra el bullying de sus alumnos, sólo el $9.9 \%$ dice no conocer a ninguno, dato que conviene relacionar con el obtenido al preguntar si conocen a algún profesor que dirija a sus alumnos este tipo de comportamientos, a lo que el $51.4 \%$ responde que conoce a uno, y el $22.7 \%$ que a tres o más. Por tanto, parece que el deterioro de las relaciones en la escuela hace que la experiencia de intimidación llegue incluso a las relaciones entre profesores y alumnos en ambas direcciones.

\subsection{VIOLENCIA, GÉNERO Y SEXISMO}

Uno de los resultados más repetidos en los estudios sobre la violencia es el de que los hombres la utilizan con mayor frecuencia y de forma más grave que las mujeres (Rutter y otros, 1998; Scrandroglio y otros, 2002), diferencia que se manifiesta desde la infancia en cualquier contexto, incluido el escolar (Olafsen y Viemero, 2000; Olweus, 1993; Whitney y Smith, 1993), y que, como sucede en otros contextos y edades, cabe relacionar con los estereotipos masculinos tradicionales, en los que se asocia el valor del hombre con el dominio, con el control absoluto y con la violencia (Cowie, 2000; Díaz-Aguado y Martínez Arias, 2001). Los análisis realizados sobre la frecuencia con la que se sufren en la escuela situaciones de víctimización en función del género, encuentran resultados menos consistentes (Olafsen y Viemero, 2000; Smith y Sharp, 1994).

Para explicar la relación entre agresión y género, conviene tener en cuenta que la principal condición de riesgo de violencia no es el sexo como condición biológica, sino la identificación con el dominio de los demás, asociado al estereotipo masculino tradicional. Esta identificación incrementa el riesgo de convertirse en agresor de los compañeros tanto en los chicos, entre los que suele ser más frecuente, como en las chicas (Young y Sweeting, 2004).

Por otra parte, en diversas investigaciones también se pone de manifiesto la necesidad de considerar diferencias cualitativas, puesto que, por ejemplo, la utilización de la violencia por las mujeres suele ser 
mucho más indirecta (Österman, 1998) y está influida por presiones situacionales con mayor intensidad (Salmivalli y otros, 1998).

En nuestro estudio (Díaz-Aguado, Martínez Arias y Martín Seoane, 2004) se encuentra, así mismo, que los chicos manifiestan en casi todos los indicadores evaluados un riesgo superior de violencia y de intolerancia que las chicas. La ausencia de diferencias de género en el ocio puede ser interpretada como un indicador de la fuerte presión grupal que suele existir en dicho contexto, que podría obstaculizar los mecanismos de inhibición de la violencia en las adolescentes, lo que refleja la necesidad de orientar la superación del sexismo de forma que favorezca una identificación con la totalidad de los valores tradicionalmente asociados a lo femenino (la empatía, la ternura) y a lo masculino (la fuerza y el poder), sin que nadie tenga que identificarse con los problemas a los que dichos estereotipos conducían (como la violencia).

\subsection{CAMBIOS SOCIALES Y RIESGO DE VIOLENCIA ESCOLAR}

Para comprender la posible influencia que los actuales cambios macrosociales de la denominada Revolución Tecnológica pueden tener en esta situación, es preciso considerar que reducen la eficacia de determinadas condiciones que hasta hace poco protegían a la infancia de la violencia adulta. Como se pone de manifiesto en algunos de los casos de violencia protagonizados en los últimos años por niños y adolescentes, que han sido ampliamente divulgados por los medios de comunicación, en losquesereflejaquereproducen guiones imposibles de inventar en dichas edades, y que disponen de una información para ejercer la violencia a la que hasta ahora en dichas edades no se tenía acceso, vemos que estos cambios afectan de una forma muy especial a los dos contextos educativos básicos tradicionales, tal como se estructuraban desde la Revolución Industrial:

- La familia nuclear, que se aisló de la familia extensa, especializándose en el cuidado de los/as hijos/as en torno a una figura, la de la madre, aislada también de lo que sucedía más allá del reducido mundo privado en el que transcurría su vida.

- La escuela tradicional, que se extendió a sectores cada vez más amplios de la población. Estuvo estructurada en torno a tres principios que parecen ser insostenibles hoy: la 
negación de la diversidad, cuya máxima expresión era la orientación de la educación a un alumno medio que nunca existió, y que excluía a quien no podía adaptarse a dicha referencia antes de llegar a la adolescencia; la obediencia incondicional al profesorado; y el currículum oculto, en función del cual se definían los papeles de profesor, de alumno, de compañero, y algunas normas no explícitas derespuesta a los conflictos, basadas en el dominio y en la sumisión, que entraban en contradicción con los valores que la escuela pretendía construir.

Para prevenir la violencia escolar es preciso adaptar los dos principales contextos educativos, la escuela y la familia, a los actuales cambios sociales, estructurando las relaciones y las actividades que en ella se producen de forma que resulten más coherentes con los valores democráticos que nuestra sociedad pretende transmitir. Existe un consenso cada vez más general izado sobre el papel que la cooperación puede tener para conseguirlo, así como sobre la necesidad de llevarla a cabo a los más diversos niveles (Ahmed y Braithwaite, 2004; Conoleyy Goldstein, 2004; Gini, 2004; J ohnson yJ ohnson, 1999; Reinke y Herman, 2002).

\section{ESTUDIOS SOBRE PREVENCIÓN DE LA VIOLENCIA ESCOLAR}

Las investigaciones realizadas sobre programas de prevención de la violencia escolar pueden agruparse en dos tipos: las que se orientan específicamente contra el acoso entre iguales, que siguen por lo común la metodología iniciada por Olweus; y las que se plantean desde una perspectiva más amplia como programas de prevención de la violencia escolar o de la violencia en general, basadas de forma habitual en el desarrollo de habilidades sociales.

\subsection{PROGRAMAS ESPECÍFICOS CONTRA EL ACOSO ENTRE IGUALES EN LA ESCUELA}

En el programa pionero desarrollado en 42 escuelas de Noruega por Olweus (1991) con 2500 alumnos de 10 a 15 años, se informaba a los centros sobre los resultados obtenidos en la evaluación de dicho problema, así como sobre la respuesta que daba el contexto, tratando de sensibilizar a profesores, a padres y a compañeros sobre la necesidad 
de no permitirlo, y creando vigilancia, normas y sanciones claras contra dicha situación. La evaluación de los resultados del programa reflejó una significativa disminución del acoso (de más del $50 \%$ ), tanto a partir de los informes de los agresores como de las víctimas, sin que las agresiones se desplazaran a otros contextos. Se observó, además, una mejoría del clima social y de la satisfacción de los alumnos con la escuela, así como una disminución general del comportamiento antisocial. Estos resultados cabe relacionarlos, por lo menos en parte, con la sensibilidad que existía en Noruega antes del programa, tras el suicidio de tres alumnos de 10 a 14 años producido después de haber sufrido el acoso de sus compañeros (Stevens, de Bourdeauhuij y van Oost, 2000).

Los intentos posteriores de alcanzar los excelentes resultados obtenidos en este primer estudio no han sido tan positivos. Como demostración cabe considerar, por ejemplo, el programa desarrollado en Bélgica (Flandes) por Stevens, de Bourdeauhuij y van Oost, (2000) con tres de los componentes básicos de la intervención contra el bullying: una política escolar de normas y de sanciones claramente contrarias al acoso; cuatro sesiones en el aula sobre estrategias y habilidades de resolución de conflictos de acoso y de apoyo a las víctimas; y el tratamiento específico con agresores y con víctimas. La comparación de los resultados obtenidos en las escuelas experimentales de primaria con las de control refleja una significativa eficacia según lo que declaran los agresores, pero no según lo que declaran las víctimas. En las escuelas de secundaria el programa no resulta eficaz a partir de ninguno de estos dos indicadores, dificultad que ya había sido detectada en otros trabajos (Smith y Sharp, 1994), y que se atribuye a lo inadecuado que puede resultar tratar de influir en adolescentes basándose en normas establecidas por los adultos. De eso se deriva la necesidad de adaptar los programas desde una perspectiva evolutiva (Stevens y otros, 2000). Las investigaciones posteriores permiten concluir que la eficacia de los resultados de los programas contra el bullying mejoran cuando se desarrollan de forma continua y desde una perspectiva de largo plazo, que como una intervención concreta (Carney y Merrell, 2001); que con frecuencia la eficacia sólo se observa en una de las dos perspectivas evaluadas, la de las víctimas (Menesini y Smorti, 1997) o la de los agresores (Stevens y otros, 2000); o que la disponibilidad de los iguales para hacer de mediadores en la resolución de conflictos y para apoyar a las víctimas es mucho menor entre los chicos (Nailor y Cowie, 1999).

Los análisis más recientes sobre la evaluación de los programas contra el acoso reflejan un creciente consenso en la necesidad de ampliar 
sus objetivos y sus actividades más allá del tratamiento específico del acoso entre iguales, para tratar de mejorar la calidad de vida en la escuela favoreciendo la cooperación a distintos niveles (Gini, 2004; Ortega, 2003).

\subsection{PROGRAMAS ESCOLARES DE PREVENCIÓN DE LA VIOLENCIA}

La revisión de los programas escolares planteada desde una perspectiva general con el objetivo de prevenir la violencia, refleja que, aunque son abundantes las publicaciones que describen pautas de intervención, son muy escasas las que suministran evidencia empírica sobre su eficacia, y menos aún las que proporcionan evaluaciones basadas en la comparación de los cambios producidos por el programa con un grupo de control. Las pocas investigaciones que analizan la eficacia de la prevención informan con cierta frecuencia de resultados de escasa relevancia (Webster, 1993; Wilson-B rewer y otros, 1991). En ese sentido se pueden interpretar, por ejemplo, las conclusiones de la revisión a las que llegaron Fields y McNamara (2003), en las que se observa que la evidencia experimental disponible hasta la fecha sobre programas de prevención primaria de la violencia llevados a cabo con adolescentes, incluyendo la comparación con grupos de control, es muy limitada. Otra carencia importante es la que tiene que ver con la ausencia de un marco teórico coherente que fundamente la intervención y que ayude a explicar los resultados, con lo cual puede considerarse también la necesidad de diseñar los programas de forma que incluyan la dimensión moral que debetener la educación contra la violencia, incrementando los valores democráticos y el sentido de comunidad (Edwards, 2001), puesto que es la conexión con los valores morales (Ortega, Sánchez y Menesini, 2002) la que guía las decisiones que permitirán utilizar las habilidades en torno a cuyo entrenamiento gira la mayoría de los programas. En tal sentido cabe destacar, por ejemplo, la eficacia comprobada en escuelas de primaria de los Programas de construcción de la paz aplicados de forma generalizada y continuada, en los que, junto al entrenamiento en resolución de conflictos, se educa en valores democráticos, de tolerancia y de respeto intercultural (Aber y Jones, 2003).

Cuando la valoración de los programas de prevención se realiza por los equipos directivos de los centros, destacan por su eficacia, en primer lugar, los que buscan cambiar la escuela para adaptarla mejor a las necesidades de los alumnos; en segundo lugar, los que promueven 
cambios y habilidades en el profesorado para prevenir la violencia; $y$, en tercer lugar, los que simplemente pretenden modificar la conducta individual de los alumnos violentos (Heerboth, 2000).

Los intentos de concretar una agenda de investigación sobre la prevención de la violencia escolar llevan a destacar la necesidad de definir cuáles son las condiciones básicas que contribuyen a promover un entorno escolar de calidad en el que no tenga cabida la violencia, implicando, a través de la cooperación, al profesorado, al alumnado y a las familias. Para lograrlo, es necesario establecer «protocolos de intervención que ayuden a conseguirlo, simplificando cuáles son sus componentes básicos» (Reinke y Herman, 2002, p. 796).

\subsection{LA COOPERACIÓN Y LA CONSTRUCCIÓN DE LA NO-VIOLENCIA COMO COMPONENTES DE LA PREVENCIÓN}

Los programas de prevención de la violencia que evaluamos en nuestro último estudio (Díaz-Aguado, 2004) giran alrededor de dos componentes básicos, que cambian la forma de definir las relaciones en las aulas: la estructuración de las actividades educativas mediante la cooperación en equipos heterogéneos, que puede llevarse a cabo a través de cualquier materia; y el desarrollo de una secuencia de 16 actividades en las que la cooperación se aplica a contenidos relacionados con el currículum de la no violencia, incluyendo problemas de acoso entre iguales, pero no sólo eso.

\section{El aprendizaje cooperativo en equipos heterogéneos}

El enfoque sociohistórico de la psicología de la actividad (Bruner, 1999; N ewman, Griffin y Cole, 1989; Vygostky, 1978) representa el marco fundamental para explicar la naturaleza de las innovaciones incluidas en nuestro programa, así como su eficacia para adaptar la escuela a las exigencias de la sociedad actual y a las características de la adolescencia. Dichas actividades suponen, respecto a los procedimientos tradicionales, dos innovaciones básicas:

- La agrupación de los alumnos en equipos heterogéneos (en rendimiento, en nivel de integración en el colectivo de la clase, en grupos étnicos, en género, en riesgo de violencia....), que ayuda a superar las segregaciones y las exclusio- 
nes que de lo contrario se producen en la escuela, a través de las cuales se perpetúan las que existen en el resto de la sociedad, y en las que se priva a los individuos de riesgo de las oportunidades necesarias para prevenir la violencia. Esta característica contribuye a luchar contra la exclusión, y a superar la desigual distribución del protagonismo que suele producirse en las aulas, que son el origen del desapego que algunos alumnos sienten hacia ellas y de muchas de las conductas de violencia.

- Un significativo incremento del poder y de la responsabilidad que se da a los alumnos en su propio aprendizaje, sobre todo en las actividades en las que se les pide que desempeñen papeles adultos como expertos en diversas áreas (medios de comunicación, prevención, política...). Por ejemplo, elaborando la Declaración de los Derechos Humanos, un decálogo para erradicar la violencia escolar, o campañas de prevención contra la violencia de género dirigidas a adolescentes. Los resultados obtenidos han permitido comprobar que ayudar a los y las jóvenes a desempeñar el papel de expertos es muy eficaz. Cuando, v. gr., hacen de expertos contra la violencia, adquieren las habilidades formales que dicha actividad supone, y se apropian al mismo tiempo de sus objetivos: la justicia, la tolerancia y el respeto mutuo.

Estas actividades favorecen el aprendizaje significativo al proporcionar un contexto social mucho más relevante que el de las actividades escolares tradicionales, y suponen la realización de tareas completas en las que se llega a una producción final (Bruner, 1999). Así, al tratar al adolescente como si fuera un profesional capaz de prevenir la violencia, y al proporcionarle el apoyo y la motivación necesarias, se favorece que pueda descubrir el significado que esta tarea tiene para el experto que habitualmente la realiza, y que llegue a identificarse con dicho significado de forma mucho más eficaz que si le pidiéramos que llevara a cabo, paso a paso, sus distintos componentes al estilo de los ejercicios de los tradicionales libros de texto: estudiar conceptos y definiciones sobre violencia, comprender los caminos que sigue un profesional de los medios de comunicación, aplicarlos a una situación hipotética, realizar individualmente los ejercicios que vienen escritos en el Manual en función de unos determinados criterios... La eficacia de las tareas completas compartidas ha sido explicada desde la psicología de la actividad, en función de su relevancia para activar la zona de construc- 
ción del conocimiento y de potenciar así el aprendizaje (Newman, Griffin y Cole, 1989).

\section{El currículum de la no-violencia}

A lo largo de investigaciones anteriores [Díaz-Aguado (dir.), 1996; 2003; Díaz-Aguado y Martínez Arias, 2001] hemos venido desarrollando una serie de tareas sobre cómo enseñar a construir los valores de igualdad, de respeto a los derechos humanos, a la tolerancia, y el rechazo al sexismo y a la violencia, que en el último trabajo [DíazAguado, (dir)., 2004] se completan con al gunas actividades dirigidas de manera específica contra el acoso entre iguales en la escuela yen el ocio, a través de una secuencia de 16 actividades básicas:

- Democracia es igualdad.

- Activación de esquemas previos y de habilidades básicas de comunicación a partir de un spot.

- Discusión sobre distintos tipos de discriminación, enfatizando la conexión con la vida cotidiana y con las discriminaciones que se producen en la escuela.

- La construcción de los derechos humanos como base de la convivencia.

- Elaboración de una declaración sobre los derechos humanos.

- Comparación con la declaración elaborada en 1948.

- Discusión sobre las violaciones de los derechos humanos en la vida cotidiana.

- Favoreciendo una representación general de la violencia que ayude a combatirla.

- Discusión sobre la naturaleza de la violencia, a partir del vídeo Odio y destrucción.

- Discusión sobre acoso y victimización.

- Racismo y xenofobia como ampliación del bloque A.

- Aprendiendo a detectar el racismo.

- Qué mala memoria tenemos. 


\section{- Sexism oyviolencia degénero.}

- Detección del sexismo y generación de alternativas.

- Discusión sobre la violencia de género a partir del vídeo Hogar, triste hogar.

- Elaboración de un mensaje para prevenir o para detener la violencia de género.

- Violencia entre iguales en la escuela y en el ocio.

- Detección de las distorsiones que contribuyen a la violencia en general.

- Detección de las distorsiones que contribuyen a la violencia entre adolescentes.

- Discusión sobre estrategias para prevenir o para detener la violencia en el ocio.

- Elaboración de un decálogo para erradicar la violencia desde las relaciones que se establecen en la escuela.

- Sesión de discusión final sobre la integración de los temas tratados con anterioridad en la propia identidad.

\section{Evaluación de la eficacia del programa}

El programa descrito fue evaluado con 783 adolescentes de centros educativos de la Comunidad de Madrid, comparando los cambios producidos en el grupo experimental con los del grupo de control, para lo cual se aplicaron, antes y después de la intervención, los siguientes instrumentos: El Cuestionario de Evaluación de la Violencia entre iguales en la Escuela y en el Ocio; el Cuestionario deActitudes hacia la Diversidad y la Violencia; y el Cuestionario sobre Estrategias de Prevención de la Violencia en el Ocio. A partir de ellos se comprobó una significativa eficacia del programa para:

- Reducir las situaciones de violencia en la escuela, especialmente las más graves, tal como son percibidas por las víctimas como por los agresores.

- Prevenir las situaciones de violencia grave en el ocio, tomando en cuenta cómo son percibidas por las víctimas y por los agresores. En ese caso se ayuda a modificar las expectativas 
pesimistas que el profesorado tiene a veces sobre la imposibilidad de prever, desde la escuela, formas de violencia que se producen fuera de ella, y cuyas principales causas suelen situarse más allá del sistema escolar.

- Reducir las situaciones de exclusión en la escuela, tal como son percibidas por las víctimas, que, cuando no se interviene, tienden a incrementarse con el tiempo.

- Desarrollar una representación de la violencia que se apoye en todo lo que pueda combatirla, y promover la tolerancia ayudando a superar las creencias que conducen a la violencia entre iguales, al sexismo y a la violencia doméstica, al racismo y a la xenofobia.

- Incrementar la disponibilidad de estrategias de prevención de la violencia en el ocio más positivas y elaboradas, rechazando el empleo de la violencia como forma de resolución de conflictos.

- Mejorar la calidad de la relación con el aprendizaje y con el profesorado, tal como es evaluada tanto por el alumnado que participa en el programa como por sus profesores. También se debe observar una tendencia encaminada a mejorar el resto de las relaciones y de los contextos sobre los que tratan las actividades del programa: la clase, los amigos, los compañeros, el instituto, el ocio.

Como muestra de cómo ve el alumnado el cambio originado por las innovaciones propuestas, incluimos a continuación extractos de las entrevistas llevadas a cabo en uno de los estudios-piloto realizados con anterioridad a la comprobación experimental que ya describimos. Manuel, J orge y J aime eran alumnos de un aula en la que, antes de llevarse a cabo el programa, la convivencia había llegado a deteriorarse de forma muy preocupante, tanto entre los propios alumnos como entre éstos y sus profesores; un aula compuesta en gran parte por repetidores, y con un al to porcentaje de estudiantes caracterizados por su conducta violenta.

MANUEL (antes de participar en el programa): «Me han echado de clase muchas veces por enfrentarme a algunos profesores [... ]. Me faltan al respeto. Abusan de ser profesores. Como el otro día, una profesora me dijo que la clase estaba mejor sin mí, y yo le contesté que si estaba mejor sin 
mí entonces también estaba mejor sin ella [...]. Si me porto mal y hay unas normas, que me las expliquen. Podrían intentar hablar conmigo, llevarme por otro camino [...] son profesores. Ellos sabrán cómo, pero no diciéndome esas cosas, porque me incitan a que me ponga nervioso y empeore todo. Esta clase es problemática. Todos Io dicen. Nos hemos juntado la mayoría repetidores [...] este año he empezado mal y no creo que tenga solución. ¿Si tú fueras director del instituto, qué harías para resolver estas situaciones? Cambiar el modo de dar la clase y echar al alumno o al profesor. Porque a mí me expulsan, pero a ellos no».

MANUEL (después del programa, en el que participó de manera intermitente debido a las expulsiones): «El ambiente de clase ha mejorado. En grupos vamos mejor. Aprendemos más, porque como hay personas que tienen más nivel, sacan mejor nota, $y$, al final, todo se acaba pegando [...]. Trabajar en clase es contagioso [...]. La relación con los profesores ha mejorado [...]. Me parece bien que se trate el tema de la violencia en clase, porque tengo el carácter muy fuerte y me enfado mucho. $Y$ si me hacen algo... me pongo nervioso. Depende de con quién me controlo o no [.. ] ya me he dado cuenta de que me puedo perjudicar; me he dado cuenta de que aquí en el instituto no sirve para nada. ¿Por qué te expulsaron? Por acumulación de partes... no me echaron porque sí. Me lo merezco. Me he dado cuenta de que por hacer el tonto voy a tener que repetir».

J ORGE (después de participar en el programa): «El ambiente en clase ha mejorado mucho. Al principio dábamos mucha guerra. Hemos mejorado, nos hemos tranquilizado. Este trimestre todo el mundo se da cuenta de que tiene que aprobar el curso, que nos pasamos mucho con los profesores [...]. Creo que el trabajo en grupos ha influido. Nos conocemos mejor, nos ayudamos en los deberes, prestamos más atención a la asignatura, las clases se hacen más divertidas [...]. Aprendemos más porque todas las dudas se resuelven entre los cuatro del grupo; no tenemos que preguntar al profesor [... ]. Las clases antes eran muy aburridas y ahora son divertidas [...]. En ética hablamos de problemas de la calle que tenemos nosotros. De la violencia en la calle, de los derechos humanos... a mi me sirve para mi vida. ¿Por qué? 
Hablamos de lo que se debe hacer y no se debe hacer, y luego, cuando se te presenta un problema en la calle, lo solucionas de otra manera. Por ejemplo, si nos pasa otra vez lo del bar (una situación de violencia entre bandas descrita al principio de la entrevista y vivida antes de tratar en clase el tema de la violencia), pasamos y nos vamos aunque se rían; nos da igual; si les plantas cara lo único que consigues es pelearte y tener más problemas; es preferible que se rían».

JIME (antes de participar en el programa): « ¿Hay situaciones de violencia en el instituto? Sí, más de una pelea ya ha pasado, pero no de pandillas, cosas aisladas. Es que este instituto es muy conflictivo. Este barrio está dividido en distintas zonas y sellevan muy mal entre ellas. Los conflictos se crean fuera del instituto pero se resuelven aquí, porque, claro, aquí nos juntamos todos, hay mucha diversidad ideológica. Racistas y todo. ¿Qué hace el instituto para resolver estos conflictos? El Instituto lo que hace es echar a algunos alumnos y ya está. En esta clase han acumulado lo peor, casi todos son repetidores y eso se nota, pero las actitudes en clase son distintas según el profesor. Hay profesores con los que no nos metemos».

J AIME (después de participar en el programa): «EI trabajo en grupos va bastante mejor. Se hace más sencillo, porque unos con otros nos podemos ayudar; en unas materias uno sabe más y ayuda al resto, y en otras al revés... ¿Qué te parece cómo se han formado los grupos? A ti te gustaría ponerte con tus amigos, pero la verdad es que están haciendo los grupos muy bien compensad os y funcionan; yo creo que ha mejorado el rendimiento del grupo. ¿Todo el mundo trabaja? Sí, y si alguien se "escaquea" se le dice algo y ya está. ¿Quién se lo dice? Los miembros del grupo. Dentro del grupo nos ponemos de acuerdo, nos organizamos el trabajo [...]. La relación con los profesores ha mejorado, y ahora trabajamos más». 


\section{CONCLUSIONES SOBRE CÓMO PREVENIR LA VIOLENCIA DESDE LAS RELACIONES QUE SE ESTABLECEN EN LA ESCUELA}

A partir de lo analizado en este artículo se desprende que, para prevenir la violencia desde las relaciones que se establecen en la escuela, es preciso:

- Adaptar la educación a los actuales cambios sociales, desarrollando la colaboración a múltiples niveles, de forma que sea posible afrontar los complejos retos sociales que la educación vive hoy. Para conseguirlo, hay que redefinir los papeles a partir de los cuales se estructura la interacción educativa, dando al alumnado un papel más activo en su propia educación, permitiendo que el profesorado incremente su autoridad, y poniendo en marcha nuevos esquemas de colaboración entre la escuela y la familia, así como con el resto de la sociedad. Estos objetivos exigen promover nuevos contextos que ayuden a la búsqueda conjunta de soluciones para una meta compartida, como es la de mejorar la educación, basados en el respeto mutuo entre los distintos agentes educativos, porque si la escuela no está hoy aislada de los problemas que se producen fuera de ella, tampoco debería estarlo para las soluciones.

- Mejorar la calidad del vínculo educativo y desarrollar el empowerment. Los estudios sobre el origen de la violencia llevan a destacar la falta de calidad del vínculo educativo como una de sus principales causas. Para prevenirla desde la escuela, es imprescindible que el profesorado desarrolle su poder de referencia, así como distribuir el protagonismo académico entre el alumnado, incrementando con ello lo que suele denominarse en distintos foros internacionales como empowerment. La escuela debe promoverlo, favoreciendo que cada alumno/a defina y desarrolle sus propios proyectos escolares. Los procedimientos educativos participativos, como el aprendizaje cooperativo en grupos heterogéneos, son de gran eficacia para conseguirlo. EI fortal ecimiento de las personas encargadas de la educación, sobre todo del profesorado, debe ser destacado también como una condición básica para mejorarla. 
- Desarrollar alternativas a la violencia en los contextos y en Ios individuos. La violencia puede ser utilizada para responder a funciones psicosociales cuando se carece de alternativas. Por eso, y para prevenirla, es preciso desarrollarlas estableciendo contextos y procedimientos alternativos en el sistema escolar (como las asambleas de aula que pueden llevarse a cabo en las tutorías) y en la familia, por medio de los cuales y de forma normalizada (sin que nadie se sienta amenazado en ellos) puedan expresarse las tensiones y las discrepancias y resolverse los conflictos sin recurrir a la violencia (através de lacomunicación, lanegpciación, la mediación. .. ), endondelasvíctim aspuedanencontrar laayuda quenecesitansinserestigm atizadesparello; yprom oviendb altemativas en todbs los individos (alumnado, profescrac...) m ediante habi lidades que perm itan afrontar la tensióny resolver los conflidos sin rearrira la violencia.

- Romper la conspiración de silencio sobre la violencia escolar, e insertar su tratamiento en un contexto normalizado orientado a mejorar la convivencia. Entre las condiciones que contribuyen a la violencia escolar, destacan tres características de la escuela tradicional: la justificación o la permisividad de la violencia entre chicos, como forma de resolución de conflictos entre iguales; el tratamiento habitual que se da a la diversidad actuando como si no existiera; y la fal ta de respuesta del profesorado ante la violencia entre escolares, que deja a las víctimas sin ayuda, y que suele ser interpretada por los agresores como un apoyo implícito. Dicha falta de respuesta está relacionada con la forma tradicional de definir el papel del profesorado, especialmente en secundaria, orientado de manera casi exclusiva a impartir una determinada materia. $Y$, como sugieren los propios profesores, podría superarse si recibieran el apoyo y la formación adecuados para afrontar el tipo de problemas que conducen a la violencia desde una perspectiva de ciudadanía democrática. En este ámbito hay que situar el establecimiento de contextos normalizados orientados a mejorar la convivencia, en los que las víctimas puedan encontrar la ayuda que necesitan y los agresores recibir una adecuada disciplina, porque, de lo contrario, la impunidad de la violencia contribuye a su incremento. 
- Enseñar a condenar toda forma de violencia, favoreciendo una representación que ayude a combatirla. Conviene orientar el rechazo a la violencia desde una perspectiva que incluya tanto su repudio de forma general, con independencia de quién sea la víctima y quién el agresor, como un tratamiento específico de sus manifestaciones más frecuentes: la violencia de género y la violencia entre iguales, ya sea en la escuela, ya en el ocio. Las medidas disciplinarias deben contribuir a lograr ese objetivo, ayudando a generar cambios cognitivos, emocionales y conductuales, que permitan que el agresor se ponga en el lugar de la víctima, que se arrepienta de haber empleado la violencia, y que intente reparar el daño originado. La eficacia de estos tres componentes mejora cuando se integran en un mismo proceso.

- Favorecer la identificación con el respeto a los derechos humanos, estimulando el desarrollo de la capacidad para ponerse en el lugar del otro, motor básico de todo el desarrollo socio-emocional, y que en sus niveles más evolucionados se extiende a todos los seres humanos, así como la comprensión de los derechos universales y la capacidad de usar esa comprensión en las propias decisiones morales, coordinando dichos derechos con el deber de respetarlos. Al incluir el rechazo a la violencia dentro de tal perspectiva conceptualizándola como una grave amenaza a los derechos humanos, se favorece su comprensión como un problema que afecta a cualquier individuo, puesto que pone en peligro el nivel de justicia necesario para que se respeten también sus derechos.

- Incluir actividades específicamente dirigidas a prevenir la vidion izacióndentro de los programas de prevención de la violencia, enseñando a decir no en situaciones que puedan implicar abuso; pedir ayuda cuando se necesite; y estar preparado emocionalmente para no sentirse culpable cuando se es víctima.

- Prevenir la intolerancia y el sexismo. Determinadas actitudes y creencias existentes en nuestra sociedad hacia los papeles y las relaciones en cuyo contexto se produce la violencia ejercen una influencia decisiva en el riesgo de ejercerla, como son las creencias racistas, sexistas y xenófo- 
bas, la conceptualización de la violencia entre iguales como una expresión de valentía, o cualquier otra creencia que lleve a rechazar a las personas que se perciben diferentes, situación en la que todos/as podemos encontrarnos. De ahí se deriva la necesidad de enseñar a detectar y a corregir dichas actitudes, como un requisito necesario para prevenir la violencia en todas sus manifestaciones.

- Educar en la ciudadanía democrática, mejorando la coherencia entre los valores que se pretenden enseñar y la práctica educativa. Uno de los principales obstáculos que debe superar hoy la educación es el que ha sido denominado currículum oculto. El incremento de los problemas de indisciplina descritos en los últimos años, sobre todo por el profesorado de secundaria, pone de manifiesto que el currículum oculto ha perdido eficacia como forma de control, y que para superar estas dificultades es necesario avanzar en la construcción de la democracia desde la escuela, una de las mejores herramientas para luchar contra la violencia y la exclusión. Por todo ello, es preciso incrementar la participación del alumnado en la construcción y en la aplicación de las normas que regulan la convivencia, y mejorar la eficacia educativa de la disciplina.

- Poner a disposición del profesorado los medios que permitan adaptar la escuela a una situación nueva. Para llevar a la práctica los principios expuestos, es preciso desarrollar condiciones que permitan a los profesores llevarlas a cabo. En tal sentido, conviene tener en cuenta la necesidad de apoyarlos, facilitando que adquieran las habilidades necesarias para conseguirlo, y creando condiciones que posibiliten la cooperación entre ellos sin caer en la frecuente tendencia a sobrevalorar sus posibilidades para desarrollar objetivos muy complejos sin los medios necesarios, ni en la tendencia contraria, infravalorando la capacidad de unos profesionales para adquirir las habilidades necesarias que permitan adaptar la educación a las exigencias de la situación actual. 


\section{BIBLIOGRAFÍA}

ABER, L., y J ONES, S. (2003): «Developmental Trajectories Toward Violence in Middle Chilhood: Course, Demographic Differences, and Response to School-based Intervention», en Developmental Psychology, 39, 2, pp. 324-348.

Ahmed,W., y Braithwalte, V. (2004): «Bullying and Victimization: Cause from Concern for both Families and Schools», en Social Psychology of Education, 7, pp. 35-54.

BAKER, J. (1998): «Are we Missing the Forest for the Trees? Considering the Social Context of School Violence», en J ournal of School Psychology, 35, 1, pp. 29-44.

BRUNER, J. (1999): La educación, puerta de la cultura, Madrid, Visor.

Carney, A., y Merrell, K. (2001): «Bullying in Schools. Perspectives on Understanding and Preventing an International Problem», en School Psychology International, 22,3, pp. 364-382.

Coleman, J. (1982): Psicología de la adolescencia, Madrid, Morata.

CONGER, J.; MILLER, W., y WALSMITH, C. (1965): «Antecedents of Delinquency: Personality, Social class and Intelligence», en: J. Mussem, J. Conger, yJ . Kagan: Readings in Child Development and Personality, Nueva York, Harper \& Row.

CONOLEY, J., y GoldSTEIN, A. (eds.): School Violence Intervention. A Practical Handbook, Nueva York, Guilford Press.

COWIE, H. (2000): «Bystanding or Standing by: Gender Issues in Coping with Bullying in Schools», Aggressive behavior, 26, pp. 85-97.

COWIE, H., y SHARP, S. (1994): «Tackling Bullying Through the Curriculum», en P. Smith y S. Sharp (eds.): School Bullying, Londres, Routledge.

Díaz-AGUADO, M. J . (dir.) (1992): Educación y desarrollo de la tolerancia, en cuatro volúmenes y un vídeo, Madrid, Ministerio de Educación y Ciencia.

- (dir.) (1996): Programas de educación para la tolerancia y prevención de la violencia en los jóvenes, en cuatro volúmenes y dos vídeos, Madrid, Instituto de la J uventud, Ministerio de Trabajo y Asuntos Sociales.

- (2002a): Convivencia escolar y prevención de la violencia, página web del Centro Nacional de Información y Comunicación Educativa, http://www.cnice.mecd.es/ recursos2/convivencia_escolar/

-. (2002 b): Prevenirla violencia contra las mujeres construyendo la igualdad, un libroy dos vídeos, Madrid, Instituto de la Mujer, Ministerio de Trabajo y Asuntos Sociales.

- (2003):Educación intercultural yaprendizaje cooperativo, Madrid, Pirámide.

- (dir.) (2004): Prevención de la violencia y lucha contra la exclusión desde la adolescencia, en tres volúmenes y dos vídeos, Madrid, Instituto de la J uventud, Los libros están también disponibles en: mtas.es/injuve/novedades/prevenciónviolencia. htm.

- (2004): Prevención de la violencia y lucha contra la exclusión desde la adolescencia, volumen dos: Programa de intervención y estudio experimental, Madrid, 
Instituto de la Juventud. También disponible en: mtas.es/injuve/novedades/ prevenciónviolencia.htm.

DíAz-A GUADO, M. J.,y MARTíneZARIAS, R. (2001): La construcción de la igualdad y la prevención de la violencia contra la mujer desde la educación secundaria, Madrid, Instituto de la Mujer, Ministerio de Trabajo y Asuntos Sociales.

Díaz-A guado, M. J.; M ARtínez ARias, R., y Martín SeOANe, G. (2004): Prevención de la violencia y lucha contra la exclusión desde la adolescencia, volumen uno: La violencia entre igual es en la escuela yen el ocio. Estudios comparativos einstrumentos de valuación, Madrid, Instituto de la J uventud.

EDWARDS, C. (2001): «Student Violenceand the Moral Dimensions of Education», en Psychology an the Schools, 38, 3, pp. 249-257. Madrid, Narcea.

FERnÁndeZ, I. (1998): Prevención de la violencia y resolución de conflictos,

Felds, S., y MCNamara, J. (2003): «The Prevention of Child and Adolescent Violence. A Review», Aggresion and Violent Behavior, 8, pp. 61-91.

GILLBORN, D. (1992): «Citizenship, race and the Hidden Curriculum», International Studies in Sociology of Education, 2, pp. 57-73.

GINI, G. (2004): «Bullying in Italian Schools. An Overview of Intervention Programs», en School Psychology International, 25, 1, pp. 106-116.

GLUECK, S., y GLUECK, E. (1960): Predicting Delincuency and Crime, Boston, Harvard University Press.

Heerboth, J. (2000): «School Violence Prevention Programs in Southern Illinois high Schools: Factors Related to Principal's and Counselor's Perceptions of Success», Dissertation

Abstracts International Section A: Humanities and Social Sciences, 60, 8-A, p. 2752.

HRITZ,S., y GABOW, P. (1997): «A peer Approach to high risk Youth», J ournal of Adolescent Health, 20,4, pp. 259-260.

HUGH-J ONES, D., y SMITH, P. K. (1999): «Self Reports of Short-and long-term Effects of Bullying on Children who Stammer», B ritish J ournal of Educational Psychology, 69, pp. 141-158.

J OHNSON, D. W., y JOHNSON, R. T. (1999): Cómo reducir la violencia en las escuelas, Barcelona, Paidós. (Fecha de la primera edición en inglés,1995).

York, Plenum Press.

LERnER, M. (1980): The Belief in a just World: A Fundamental Delusion, Nueva

MendozA, B. (2005): La otra carade la violencia. El maltrato en la interacción profesor-alumno, Madrid, Universidad Complutense (informe de investigación inédito).

MENESINI, E., y SMORTI, A. (1997): «Strategie d'intervento scolastico» en A. Fonzi (ed.), II bullism in Italia, Florencia, Giunti. 
NABUZOKA, D., y S MITH, P. (1993): «Sociometric Status and Social Behavior of

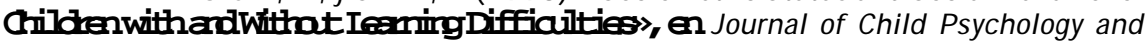
Psychiatry, 34, pp.1435-1448.

NAILOR, P., yCOWIE, H. (1999): «The Effectiveness of peer Support Systems in Challenging School Bullying: The Perspectives and the Experience of Teachers and Pupils», en J ournal of Adolescence, 22, 4, pp. 453-459.

NeWman, D.; Griffin, P., y Cole, M. (1989): The Construction zone: Working for Cognitive Change in School, Cambridge, Cambridge University Press (traducción al castellano en Morata).

OLAFSEN, R., yVIEMERO, V. (2000): «Bully/victim Problems in Coping with Stress», en School Among 10 -to1 2 Years old Pupils in Åland, Finland. Aggressive Behavior, 26, pp. 57-65.

OLWEUS, D. (1991): «B ully-victim Problems Among School Children. Basic Facts and Effects of a School Based Intervention Program», en D. Pepler; K.. Rubin (eds.): The Development and Treatment of Childhood Agression, Hillsdale, N. J ., Lawrence Erlbaum.

OLWEUS, D. (1993): Conductas de acoso y amenaza entre escolares, Madrid, Morata, 1998. (Fecha de la edición en castellano).

ORTEGA, R. (2003): «El Proyecto Antiviolencia escolar: Andave», en Boletín Colegio Oficial de Doctores y Licenciados, pp. 17-23.

ORTEGA, R.; SÁnCHEZ, V., y MENESINI, E. (2002): «Violencia entre iguales y desconexión moral: un análisis transcultural», en Psicothema, 14, pp. 37-49.

ÖSTERMAN, D.; B J ÖRKQVIST, D., y LAGERSPEZT, K. (1998): «Cross-cultural Evidence of Female Indirect aggression», en Aggressive Behavior, 24, pp. 1-8.

Pellegrini, A.; B ARTINI, M., y BRooks, F. (1999): «School Bullyes, Victims and Aggressive Victims», en J ournal of Educational Psychology, 91, pp. 216-224.

ReINKE, W., y HERMAN, K. (2002): «A Research Agenda for School Violence Prevention», en American Psychologist, 57,10, pp. 796-797.

RIVERS, I. (1999): «Peer Victimisation and life-span Development», en Paper presented at the Inaugural European Conference on Gay and Lesbian Identities, U niversity College, Londres, marzo (citada por Eslea y Rees, 2001).

RUTTER, M.; GILler, H., y HAGEL, A. ( 1998): Antisocial B ehavior in Young People, Cambridge, Cambridge University Press.

SALMIVALLI, C. (1999): «Participant role Approach to School Bullying: Implications for Intervention», Journal of Adolescense, 2, 4, pp. 453-459.

Salmivalli, C.; Lagerspetz, M.; BJ ÖRkQvist, K..; Österman, D., y Kauklainen, A.. (1996): «Bullying as a Group Process», en Aggressive Behavior, 22, pp. 1-15.

Salmivalli, C.; L APPALAINEN, M:, y LAGERSPETZ, M. (1998): «Stability and Change of Behavior in Connection with Bullying in Schools: A two year Follow up», en Aggressive Behavior, 24, pp. 205-218. 
Sandroglio, B.; Martínez, J.; Martín, M. J i óPez, J.; M ARTín, A.; S AN J Osé, M. C., y MARTín, J. (2002): «Violencia grupal juvenil: una revisión crítica», en Psicothema, 14, pp. $6-15$

SCHWARTZ, D.; DODGE, K.; P ETTIT, G., y BATES, J . (1977): «The Early Socialization of Aggressive Victims», en Child Development, 68, 4, pp. 665-675.

SMITH, P., yS HARP, S. (eds.) (1994):School Bullying. Insights and Perspectives, Nueva York, Routledge.

SMith, R. ; TAlamelli, L.; COWIe, H.; Naylor, P., y Chauhan, P. (2004): «Profiles of non-victims, Escaped Victims, Continuing Victims and new Victims of School Bullying», en British J ournal of Educational Psychology, 74, pp. 565-581.

STEVEnS, V.; De BourdeaudhuIJ, I., y VAN OOST, P. (2000): «Bullying in Flemish Schools: An Evaluation of Antibullying Intervention in Primary and Secondary Schools», en British J ournal of Educational Psychology, 70, pp. 195-210.

TERRY, A. (1998): «Teachers as Targets of Bullying by Their Pupils: a Study to Investigate Incidence», en British J ournal of Educational Psychology, 68, pp. 255-268.

TRIANES, V. (2000): La violencia en contextos escolares, Málaga, Aljibe.

TORREGO, J . C., y MORENO, J. M. (2003): Convivencia y disciplina en la escuela. El aprendizaje de la democracia, Madrid, Alianza.

TroYnA, B., yHATCHER, R. (1992): Racism in Children's Lives: A Study of Mainly White Primary Schools, Londres, Routledge.

VYGOTSKY, L. (1978): Mind in Society. M. Cole; V. J ohn Steiner; S. Scribner, y E. Soubermen (eds.), Cambridge, Harvard University Press.

Webster, D. (1993): «The Unconvincing case for School-based Conflict Resolution Programs for Adolescents», en Health Affairs 12 (4) pp. 126-140.

WHITNEY, I.; N ABUZOKA, D., y SMITH, P. (1992): «Bullying in Schools: Mainstraim and Special Needs», en Support for Learning, 7,1, pp. 3-7.

WHITNEY, I., y SMITH, P. (1993): «A Survey of the Nature and Extend of Bullying in J unior/middle and Secondary Schools», en Educational Research, 35, pp. 3-25.

WILSON-BREWER, R.; COHEN, S.; O'DONNELL, L., y GOODMAN, I. (1991): «Violence Prevention for Young Adolescents: A Survey of the State of the Art» (Eric Clearinghouse, ED356442, 800-443-3742).

Young, R., y SWEETING, H. (2004): «Adolescent Bullying, Relationships, Psychological well-being, and Gender-atypical Behavior: A Gender Diagnosticity Approach», en Sex Roles, 50, 7-8, pp. 525-555. 


\title{
REVISTA IBEROAMERICANA DE EDUCACIÓN
}

\author{
REGRESAR A ÍNDICE № 37
}

REGRESAR A PÁGINA INICIAL DE LA REVISTA

CONTACTAR 